\title{
New approach to spectral features modeling
}

\author{
Hedser van Brug* and Paolo S. Scalia \\ TNO Delft, Stieltjesweg 1, 2628 CK Delft, the Netherlands
}

\begin{abstract}
The origin of spectral features, speckle effects, is explained, followed by a discussion on many aspects of spectral features generation. The next part gives an overview of means to limit the amplitude of the spectral features. This paper gives a discussion of all means to reduce the spectral features amplitude (SFA), i.e. inner pixel averaging, coherence effects, and angular averaging, and to a lesser extend coherence effects and polarization. A new approach to model spectral features is presented, that is based on the averaging mechanisms, in a similar way as is used for speckle patterns. Formulae for all averaging methods are given and the use is discussed. The paper starts off with a short description on Spectral Features as observed in earth observation spectrometers, followed by speckle effects and averaging mechanisms. Next the origin of the averaging mechanisms in a spectrometer are given and a method to calculate them.
\end{abstract}

Keywords: Radiometric accuracy, Speckles, Spectral Features, Remote Sensing

\section{INTRODUCTION}

Earth observing satellites for spectroscopic analysis of the earth atmosphere take the sun as illumination unit. The spectra recorded after passage through the atmosphere are calibrated using direct sun observations. To mimic the scattering of the earth, an on-board diffuser is included in the satellites. An additional function of this diffuser is to get a homogeneous illumination of the entrance slit of the spectrometer and to monitor the aging of the instrument. A final task of the diffuser, or better put, a further requirement for the diffuser is that it should diffuse the light to that extend that the radiometric throughput is in the allowed range.

In the past years a number of reports ${ }^{1,2}$ have been made about wiggles that are observed in the calibrated spectra of earth observation satellites that can not be due to absorption in the atmosphere. In the 2004 SPIE annual meeting a complete session was dedicated to the discussion of these wiggles. During that meeting we presented our research ${ }^{3}$ in this field. We have created a setup that is designed for testing diffusers and to get insight in the means to minimize the wiggle amplitudes. A model to describe the wiggles was presented and results obtained using this model were discussed. Now we will repeat the basics of the model and give an extensive description of the results of the model and the experimental results obtained using our setup. All presently known methods that can be used to reduce the amplitude of the wiggles will be presented.

In order to have a simple quantity to relate to a given diffuser, that tells directly the amplitudes of the wiggles that are to be expected, we propose the use of the Spectral Features Amplitude (SFA). Spectral Features are those features in the spectra that are due to the diffuser (and are not due to absorption effects in the earths atmosphere or due to emission lines in the spectrum of the light source). The standard deviation of the normalized data is taken as the SFA. The SFA can be calculated over a spectral width containing a few periods of spectral features. Alternatively, the SFA can be calculated from a large number of independent spectra where the standard deviation is calculated per wavelength. Differently stated the Spectral Features Amplitude can be defined as: the deviation of the unwanted wiggles in the signal as measured in a spectrometer after eliminating all wanted features from the spectrum, e.g. by dividing two independent measurements.

*E-mail: eddy.vanbrug@tno.nl; Phone: +31 888668055

Earth Observing Systems XVII, edited by James J. Butler, Xiaoxiong Xiong, Xingfa Gu,

Proc. of SPIE Vol. 8510, 85100G · @ 2012 SPIE · CCC code: 0277-786X/12/\$18 · doi: 10.1117/12.931497 


\section{CAUSE OF SPECTRAL FEATURES}

When laser light (coherent or at least partially coherent $\left.{ }^{4}\right)$ is scattered by a rough surface speckles are observed. This is due to interference effects that occur in all locations of an observation screen. Each location on that screen receives light from many points on the scattering object. All contributions can interfere as long as the coherence length of the light source is long enough such that path length differences are smaller than this coherence length.

In case of an earth observation satellite the light source in use is the sun. The coherence length of the light emitted by the sun is very short such that speckle effects are not easily observed. However, since the spectrometer limits the wavelength band per pixel, the light reaching each pixel will show interference effects and thus also speckles. The effective coherence length $\mathrm{L}_{\mathrm{c}}$ for a spectral width of $\Delta \lambda$ and a central wavelength of $\lambda_{0}$ is obtained via

$$
L_{c}=\frac{\lambda_{0}^{2}}{\Delta \lambda} \text {. }
$$

The number of speckles present on each pixel changes with distances in the setup, illumination angle of the diffuser, etc. This means that the observed intensity per pixel will change over time. As a function of wavelength the intensity will even change for a perfectly stable setup. These intensity fluctuations with wavelength, that are due to changing speckle numbers and/or intensities, are what we refer to as Spectral Features. These Spectral Features cannot be cancelled via a calibration scheme since they change with about everything. In a laboratory setup calibrating out the Spectral Features is feasible but in an operational satellite this is not an option.

The speckle size $\sigma_{\mathrm{s}}$ scales linearly with wavelength,

$$
\sigma_{s}=0.61 \frac{\lambda}{\mathrm{NA}}
$$

which means that the number of speckles falling onto a one dimensional pixel scales inversely proportional to the wavelength. The effect of the increase or decrease of the number of speckles in the detection area by one is stronger for a few speckles that when many speckles are present. This means that the Spectral Features will be more pronounced for longer wavelengths. The NA in Eq.(2) stands for numerical aperture of the optical system. In the case that a lens is used in the system the NA is defined by the aperture of the lens and the distance between lens and observation plane (in this paper this is taken to be the entrance slit of the spectrometer). If no lens is used, free space propagation, the NA is defined by the width of the scattering surface and the distance between diffuser and observation plane.

The following section gives a step-wise description of the modeling approach.

\section{SPECTRAL FEATURES MODELING}

To model the spectral features a fixed approach is followed. We start with determining the so called intrinsic SFA, the SFA as would be obtained in the absence of all averaging mechanisms. This intrinsic SFA follows from the simulation tool but can, as a check to see if all characteristics have been implemented correctly, also be calculated from speckle theory. The intrinsic SFA is then defined as the speckle pattern contrast of a speckle pattern on a detector pixel.

\subsection{Determination of intrinsic SFA}

The determination of the spectral features is based on the definition of the complex field (and hence the speckle pattern) in the spectrometer. This complex field results in the description of the intensity distribution on the sensor. The spectral features amplitude is then obtained by taking the ratio of several intensity distributions.

In general the optical setup of a calibration unit will consist of several optical elements such as some diffuser plates (separated by a spacer), a lens and a focal plane with spectral pixels. Using the simulation tool, in between the optical elements the field is propagated, and subsequently the field at each interface is determined.

The calculation is started at the diffusers. Each point on the rough surface will scatter the incoming light over a certain opening angle. At any distance $d$ from the scattering surface one can calculate the resulting light intensity by coherently 
adding the contributions from all scatter locations on the surface. The distance between the observation point and each scattering location has to be taken into account. If more than a single scattering surface is present this approach is repeated until the final element has been reached. From that point onwards three propagation modes exists: free propagation, imaging and Fourier mode.

Free propagation means that there are no elements with optical strength between two calculation planes. In the case of imaging the starting plane is imaged onto the end plane. This step can be performed by calculating the convolution of the incoming field with a spherical wave. The final mode is used in case the starting and end plane are the two focal planes of a lens or mirror.

Using this approach the intensity on a pixel is calculated as a function of wavelength. By changing the roughness of the diffusers or the angle of incidence, an uncorrelated speckle patterns is obtained. By determining the ratio between two uncorrelated spectra the SFA is obtained by calculating the standard deviation of the thus obtained spectrum. This SFA value is the square root of two larger than the speckle contrast of a detector pixel. By comparing these values the implementation of the system into the simulation tool can be checked.

\section{AVERAGING MECHANISMS}

The intrinsic SFA has to be scaled by the effect of the averaging mechanisms to be obtain the observed SFA. This section gives an overview of the mechanisms to be taken into account and how they will affect the intrinsic SFA. It is assumed that the spectral features amplitudes are constant over the range on which the averaging is carried out, so that averaging effectively becomes a multiplication of the spectral feature amplitudes by a constant:

$$
S F A=S F A_{\text {intr }} \cdot F
$$

where the averaging mechanisms, indicated as $F$, depend on the parameters over which is averaged. Note that, although denoted here as a constant, the value of $F$ may depend on the several parameters, such as the wavelength.

The averaging mechanism is effectively a reduction of the amplitude of a noise signal, which can be reduced by averaging over $N$ uncorrelated measurements, resulting in a reduction by the square root of $N$. This approach can be found in literature on speckle statistics and ways to reduce the speckle contrast.

\subsection{Angular averaging}

The opening angle of the sun illuminating the diffuser is about 0.5 degrees, which means that angular averaging will always be present. The correlation between two spectra, simulated for different angles of incidence, yields information on the minimum angular change needed to arrive at independent spectra. The ratio between the opening angle of the illumination $\left(\theta_{\text {sun }}\right)$ and this minimum angular change $\left(\theta_{\text {corr }}\right)$ is the SFA reduction factor owing to the angular effect.

$$
S F A \propto S F A_{\text {intr }} \frac{\theta_{\text {corr }}}{\theta_{\text {sun }}} .
$$

The SFA as a function of angle, calculated as the ratio between two spectra, one the initial spectrum, and the second the spectrum at ever increasing angular change with respect to the angle for which the first spectrum was fitted to the following function, (an empirical formula):

$$
S F A \propto S F A_{\text {intr }}\left(1-e^{-\theta / \theta_{\text {corr }}}\right) .
$$

Equation (5) has been fitted to measured data. From the fit the intrinsic SFA and $\theta_{\text {corr }}$ were obtained. Using these experimental results it was found that $\theta_{\text {corr }}$ can also be obtained by calculating the correlation length of the intensity for a single wavelength as a function of angle. 


\subsection{Inner pixel averaging}

In this section the effect of dispersion on the SFA is evaluated and estimates are given for several distinct cases. These cases will be treated differently as follows.

\section{Case 1: $\Delta \lambda_{\text {corr }}>\Delta \lambda_{\text {pixel }}$}

The spectral correlation length is larger than the bandwidth seen by a detector pixel. In this case the effect of dispersion would be a smearing out of the speckle pattern in the spectral direction. This smearing out will result in a contrast reduction equal to the effective width $\left(P_{x}\right)$ of a detector pixel, determined by the slit width, divided by the speckle width $\left(\sigma_{s}\right)$. It follows that the inner pixel averaging term in Eq.(3) becomes

$$
S F A \propto S F A_{\text {intr }} \sqrt{\frac{\sigma_{s}}{P_{x}}} .
$$

The ratio $P_{x} / \sigma_{s}$ in Eq. (6) gives the number of independent speckle patterns that contribute to the lowering of $S F A_{\text {intr }}$.

Case 2: $\Delta \lambda_{\text {corr }} \ll \Delta \lambda_{\text {pixel }}$

Case 2 handles the situation where the spectral correlation length is much smaller than the bandwidth seen by a detector pixel

where

$$
\frac{\Delta \lambda_{\text {corr }}}{\Delta \lambda_{\text {pixel }}} P_{x}<\sigma_{S}
$$

$$
\Delta P_{x} \equiv \frac{\Delta \lambda_{\text {corr }}}{\Delta \lambda_{\text {pixel }}} P_{x}
$$

indicates the speckle displacement with respect to $P_{x}$, induced by dispersion.

In this case the smearing effect due to dispersion is less than a speckle width and can therefore be neglected. This averaging mechanism is active within a single pixel and its effectiveness is due to the fact that many speckles fit within a single pixel. In this case the inner pixel averaging term in Eq.(3) becomes

$$
S F A \propto S F A_{\text {intr }} \sqrt{\frac{\Delta \lambda_{\text {corr }}}{\Delta \lambda_{\text {pixel }}}} .
$$

The main difference between the two scaling effects is that the first (Case 1) scales with the number of speckles per pixel width, whereas the second (Case 2) scales with the number of spectral correlation lengths that fits in the wavelength range as seen by a pixel. 


\section{Case 3: Intermediate case}

In general, $\Delta \lambda_{\text {corr }}$ will be between the two limiting cases that are described above. Suppose that the shift $\Delta P_{x}$ is smaller than a pixel size $P_{x}$ but larger than $\sigma_{s}$. The total averaging effect that lowers the contrast is then due to a combination of the effects described by case 1 and 2 , and is given by

$$
S F A \propto S F A_{\text {intr }} \sqrt{\frac{\sigma_{s}}{\Delta P_{x}}} \sqrt{\frac{\Delta \lambda_{\text {corr }}}{\Delta \lambda_{\text {pixel }}}}=S F A_{\text {intr }} \sqrt{\frac{\sigma_{s}}{\frac{\Delta \lambda_{\text {corr }}}{\Delta \lambda_{\text {pixel }}} P_{x}}} \sqrt{\frac{\Delta \lambda_{\text {corr }}}{\Delta \lambda_{\text {pixel }}}} .
$$

Note that since the square root terms describe averaging mechanisms they cannot be larger than unity. In case they become larger than unity that averaging is no longer effective. The square root terms have to be inspected independently, i.e. the corresponding terms can in general not cancel each other. In Case 2 the first square root will become larger than unity due to the fact that the shift in the speckle pattern due to dispersion will be smaller than the speckle size. This automatically gives a limit for which the smearing effects have to be taken into account, i.e.

$$
\frac{\Delta \lambda_{\text {corr }}}{\Delta \lambda_{\text {pixel }}} P_{x}<\sigma_{s}
$$

i.e. as soon as the shift due to dispersion is equal or smaller than the speckle width the averaging owing to the dispersion become negligible and Eq.(8) will reduce to Eq.(7). In the limit of Case 1 the second term becomes unity and Eq.(8) reduces to Eq.(6).

\subsection{Polarization averaging}

Polarization averaging is based on the effect that perpendicular polarized beams cannot interfere. This means that two orthogonally polarized independent speckle patterns will be present on the detector. This effect gives a square root of two reduction of the SFA. In formula this is:

$$
S F A \propto S F A_{\text {intr }} \frac{1}{\sqrt{2}} .
$$

\subsection{Coherence averaging}

Coherence averaging is due to the coherence length of the light. This mechanisms plays a role when contributions from scattering elements longitudinally farther apart than the coherence length of the light are present. Due to this separation these contributions cannot interfere and will result is an SFA reduction by one over the square root of the number of independent contributions.

$$
S F A \propto S F A_{\text {intr }} \frac{1}{\sqrt{\text { Coh }_{\text {aver }}}} .
$$

This averaging mechanism is virtually not present in case of a diffuser arrangement consisting purely of transmission diffusers since the contribution from the direct transmitted light will be at least an order of magnitude higher than the contributions from the multiply reflected components. In case of reflective diffusers coherence averaging will be effective. For a QVD the reflection from the first rough surface will form a speckle patterns that is uncorrelated with the speckle patterns that is formed by the light that is transmitted through the front surface and reflected by the aluminum scattering surface on the back of the QVD. In case of a Spectralon type of diffuser the thickness has to be divided by the correlation length (inside the material) to arrive at the number of independent speckle patterns. 


\subsection{Sun measurements time averaging}

During the sun measurements time the incidence angle of the sun will change. The effective opening angle will thus increase in one direction and will result in an averaging effect which can be written as

$$
S F A \propto S F A_{\text {intr }} \sqrt{\frac{\theta_{\text {sun }}}{\theta_{\text {scan }}}}
$$

where $\theta_{\text {scan }}$ is the change in angle of incidence. However, due to the small angular movement of the sun during a recording, in the order of the opening angle of the sun, this averaging mechanism will in general be very ineffective. The averaging as described in Eq.(12) was shown to be correct for a surface diffuser (e.g. a piece of aluminum with high roughness). For a diffuser with more than one scattering surface, such as a QVD, the reduction by a change in incidence angle will be stronger but at this time the amount by which it will be better is not known. Experiments on QVD show that the integration time averaging does not scale by the solar angle $\theta_{\text {sun }}$ but rather by the angular change required to arrive at an independent spectrum in case of collimated light $\theta_{\text {corr }}$. For QVD it seems to be the case that the second rough surface removes the limiting effects on averaging due to the opening angle of the source, i.e.

$$
S F A \propto S F A_{\text {intr }} \sqrt{\frac{\theta_{\text {corr }}}{\theta_{\text {scan }}}}
$$

This topic requires further analysis.

In the present model no interference effects are taken into account. It is expected that interference that can occur owing to the partial spatial coherence is responsible for the angular averaging behavior during sun measurements. To investigate a strongly different model has to be created in which the simulations are no longer based on collimated beams. The new model should start with the definition of an extended sources with adjustable characteristics in terms of spatial coherence. At present it is believed that the sun measurements time averaging as described by Eq.(13) is valid for all types of diffusers. It is expected that the averaging as given by Eq.(12) was erroneously formulated due to the fact that the angular correlation length in case of aluminum is about equal to the opening angle of the sun. More research into this topic is required.

\section{DISCUSSION AND CONCLUSIONS}

The approach as described in this paper consists of the following steps:

Calculation of the intrinsic SFA based on the ratio between two uncorrelated spectra or from the speckle contrast on a detector pixel. This latter method is easier but the first approach is readily available since these spectra have to be simulated anyway.

The next step is to determine the determine the angular dependence. To get this number spectra are simulated for many angles of incidence. For all wavelengths the intensity can now be plotted as a function of angle of incidence. The auto correlation of this plot gives the angular correlation length.

From the same data set the wavelength correlation length can be determined by calculating the auto correlation of the spectra for a given angular setting. This wavelength correlation length has to be compared with the detector bandwidth. If the wavelength correlation length is in the same order as the detector bandwidth or larger the speckle patterns will be smeared out due to the dispersive element in the spectrometer and only the averaging based on the speckle size be important. For a very small wavelength correlation length the ratio between bandwidth and correlation length determines the averaging power.

The polarization and coherence averaging can be applied based on simple scaling laws. The polarization effect is always the square root of two while the coherence averaging can be approximated by determining the number of independent speckle patterns due to extra reflections. 
We have presented a straight forward method to simulate the spectral features amplitude. Following the presented steps will lead to an accurate estimate of the to be expected relative radiometric accuracy of the spectrometer in earth observation satellites.

\section{REFERENCES}

[1] A. Richter, F. Wittrock, A. Ladstätter-Weißenmayer, J. Burrows "GOME measurements of stratospheric and tropospheric BrO", Advanced Space Research 29 (11), 1667-1672 (2002)

[2] B. Ahlers, G. Bazalgette Courrèges-Lacoste, C. Schrijvers, and H. van Brug, "In-orbit detection of Spectral Features in SCIAMACHY", In: Sensors, Systems, and Next-Generation Satellites VIII, Proc. SPIE 5570, Maspalomas, Spain, September 13-16, pp.401-410 (2004)

[3] H. van Brug, R. Vink, J. Groote Schaarsberg, G. Bazalgette Courrèges-Lacoste, and B. Snijders, "Speckles and their effects in spectrometers due to on-board diffusers", In : Earth Observing System IX, Proc. SPIE 5542, Denver, Colorado USA, August 2-6, pp. 334-341(2004)

[4] H. Fujii and T. Asakura, A contrast variation of image intensity under illumination of partially coherent light", Optics Communications 12 (1), 32-38 (1974) 